\title{
N-a-acetyltransferase 10 (NAA10) in development: the role of NAA10
}

\author{
Mi-Ni Lee ${ }^{1}$, Hyae Yon Kweon ${ }^{1}$ and Goo Taeg Oh
}

\begin{abstract}
$\mathrm{N}$-a-acetyltransferase 10 (NAA10) is a subunit of $\mathrm{N}^{a}$-terminal protein acetyltransferase that plays a role in many biological processes. Among the six N-a-acetyltransferases (NATs) in eukaryotes, the biological significance of the $\mathrm{N}$ terminal acetyl-activity of Naa10 has been the most studied. Recent findings in a few species, including humans, indicate that loss of N-terminal acetylation by NAA10 is associated with developmental defects. However, very little is known about the role of NAA10, and more research is required in relation to the developmental process. This review summarizes recent studies to understand the function of NAA1O in the development of multicellular organisms.
\end{abstract}

\section{Introduction}

$\mathrm{N}-\alpha$-acetyltransferase 10 (NAA10), the catalytic subunit of $\mathrm{N}$-acetyltransferase A (NatA), a major N-terminal acetyltransferase complex, catalyzes the alpha (N-terminal) acetylation of nascent peptides as a cotranslational modification and epsilon (internal) acetylation of mature proteins (including itself) as a posttranslational modification $^{1-4}$. NAA10, which is conserved from yeast to humans and expressed in most cell types, is an important regulator in diverse biological processes, such as cell growth, differentiation, metastasis, apoptosis, and autophagy $^{4-10}$. In mammalian cells, most studies about the function of NAA10 are mainly focused on its relationship with cancer. Meanwhile, the biological significance of NAA10 is not as well understood, and further studies are needed in the context of a developing multicellular organism. Recently, NAA10 has been reported to play a critical role in development and human genetic diseases ${ }^{11-16}$. According to these reports, several human genetic diseases have been shown to be associated with NAA10 mutations, thus highlighting the importance of NAA10 function during biological development. In this

\footnotetext{
Correspondence: Goo Taeg Oh (gootaeg@ewha.ac.kr)

${ }^{1}$ Immune and Vascular Cell Network Research Center, National Creative Initiatives, Department of Life Sciences, Ewha Womans University, Seoul, Republic of Korea

These authors contributed equally: Mi-Ni Lee, Hyae Yon Kweon
}

review, we mainly focus on NAA10 function during the development of multicellular organisms.

\section{Expression of NAA10 during embryonic development}

NAA10 is located on chromosome $\mathrm{Xq} 28$ in humans and $\mathrm{X}$ A7.3 in mouse, and is encoded by 8 exons ${ }^{17,18}$. Alternative splicing of its mRNA produces several isoforms of NAA10. There are three mouse variants $\left(\mathrm{mNaa10}{ }^{198}\right.$, $\mathrm{mNaa} 10^{225}$, and $\mathrm{mNaa} 10^{235}$ ) and two human variants $\left(\mathrm{hNaa} 10^{131}\right.$ and $\mathrm{hNaa} 10^{235}$ ). The $\mathrm{mNaa} 10^{225}, \mathrm{mNaa} 10^{235}$, and $\mathrm{hNaa} 10^{235}$ are the functional isoforms that contain the full $\mathrm{N}$-acetyltransferase domain sequence, and they have been the most extensively studied and characterized among the aforementioned variants ${ }^{18-20}$. Additionally, a homologous gene of $N A A 10$, termed NAA11, has been identified. The human NAA11 is located on chromosome $4 \mathrm{q} 21.23$, and the sequence of hNAA11 protein is $81 \%$ identical to hNAA10, whereas mouse Naa11 is on chromosome $5 \mathrm{E} 3^{21}$.

Previous studies showed the broad and ubiquitous expression of NAA1O in various cell types, including tumor cell lines and several tissue types, in the developmental stages of embryos and in adults. In general, dynamic changes in the spatiotemporal expression of distinct genes are shown during embryogenesis ${ }^{22}$. The regulation of tissue- and stage-specific expression affects the development of different organs individually ${ }^{23}$. On the 


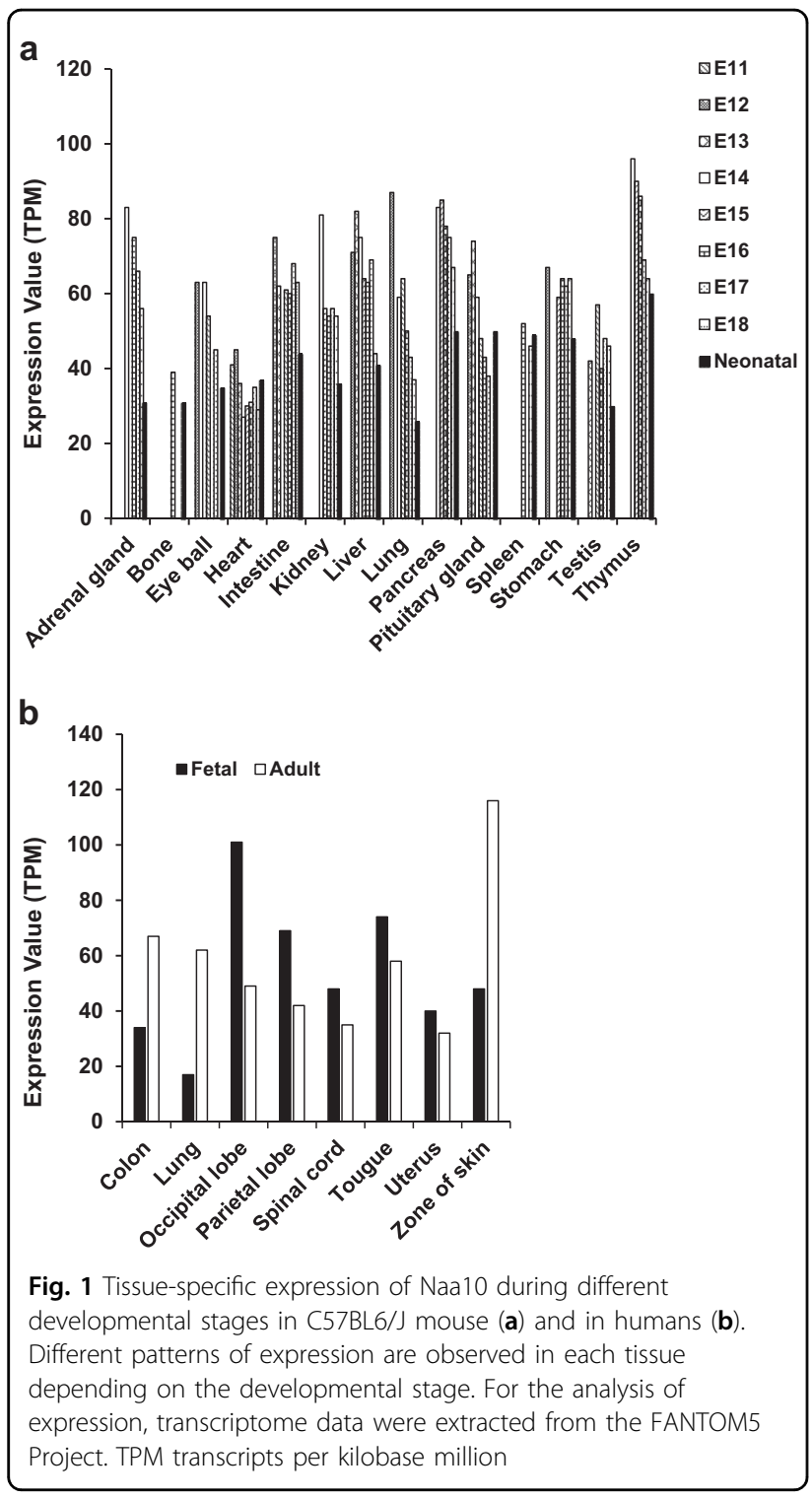

basis of this information, it is reasonable to suggest that the potential role of NAA1O varies depending on transcriptional levels in different tissues and embryonic stages during development. Interestingly, in mouse, according to expression atlas databases, the RNA expression level of $m N a a 10$ for each organ is higher in the developmental stages of embryo than in the neonate ${ }^{24,25}$. For example, in kidney, liver and lung, the RNA level of $m N a a 10$, which is very high in the stage from E12 to E14, decreased to half of this level in the neonate (Fig. 1a). A broad comparison of RNA levels between fetus and adult in humans indicated the same results as those found for mouse $\mathrm{e}^{24-27}$ (Fig. 1b). However, there are other instances in which an increased or steady expression level is shown in some tissues at the postnatal stage, so we cannot form a definitive conclusion. Whether the changes in tissue- and stage-specific expression have an impact on development needs further investigation.

\section{$\mathrm{N}$-terminal acetylation dependent functions of NAA10 during embryonic development}

Acetylation is one of the protein modifications that diversifies the function of genes in organisms. In particular, N-terminal acetylation occurs in $80 \sim 90 \%$ of eukaryotic proteins, and the molecular role of $\mathrm{N}$-terminal acetylation affects various protein functions, including protein interaction, localization to the ER and degradation. To date, six distinct forms of NATs (NatA - NatF) have been identified in eukaryotes, and they are classified based on different subunit compositions ${ }^{28,29}$. Among others, NatA undertakes the majority of $\mathrm{N}$-terminal acetylation, acetylating $\sim 40 \%$ of the human proteome $\mathrm{e}^{10,28-31}$. However, the distinct function of NatA has not yet been demonstrated during embryonic development. Nonetheless, abnormal phenotypes were observed in several species due to the absence or reduction of NAA10. These data are of great interest for understanding the role of NAA10 in development.

\section{Developmental roles in humans}

Recently, the importance of NAA10 catalytic activity in human development has emerged through the discovery of various NAA10 mutations in several pathological conditions (Table 1). Rope et al. reported the first human genetic disorder caused by a Ser37Pro mutation in NAA10 and termed the lethal X-linked Ogden syndrome $^{14,32}$. Since this discovery, more human genetic disorders caused by NAA10 missense mutations, including Tyr43Ser, Arg83Cys, Phe128Leu/Ile, Val107Phe, and Arg116Trp, have been discovered ${ }^{11,13,15,16}$. Among a wide spectrum of malformations in NAA10-related syndromes, there are some common phenotypes with global developmental delay, cardiac anomalies, and intellectual disabilities, and the corresponding NAA10 mutations triggered a reduction in catalytic activity, suggesting that NATs are essential during early embryogenesis and that they possess important regulatory functions during tissue and organ development ${ }^{11,33}$.

Additionally, a splice mutation in the intron 7 splice donor site $($ c. $471+2 \mathrm{~T} \rightarrow \mathrm{A})$ of NAA10 was implicated as a cause of Lenz microphthalmia syndrome ${ }^{12}$. Developmental abnormalities, such as microphthalmia or anophthalmia, developmental delay, intellectual disability, skeletal abnormalities and malformations of teeth, fingers, and toes, were observed. Gene expression array studies in patient fibroblasts displayed the dysregulation of several genes involved in embryonic, organ, tissue and skeletal and muscular system development, and above all, these changes were associated with the retinoic acid and Wnt signaling pathways, which are necessary for normal eye 


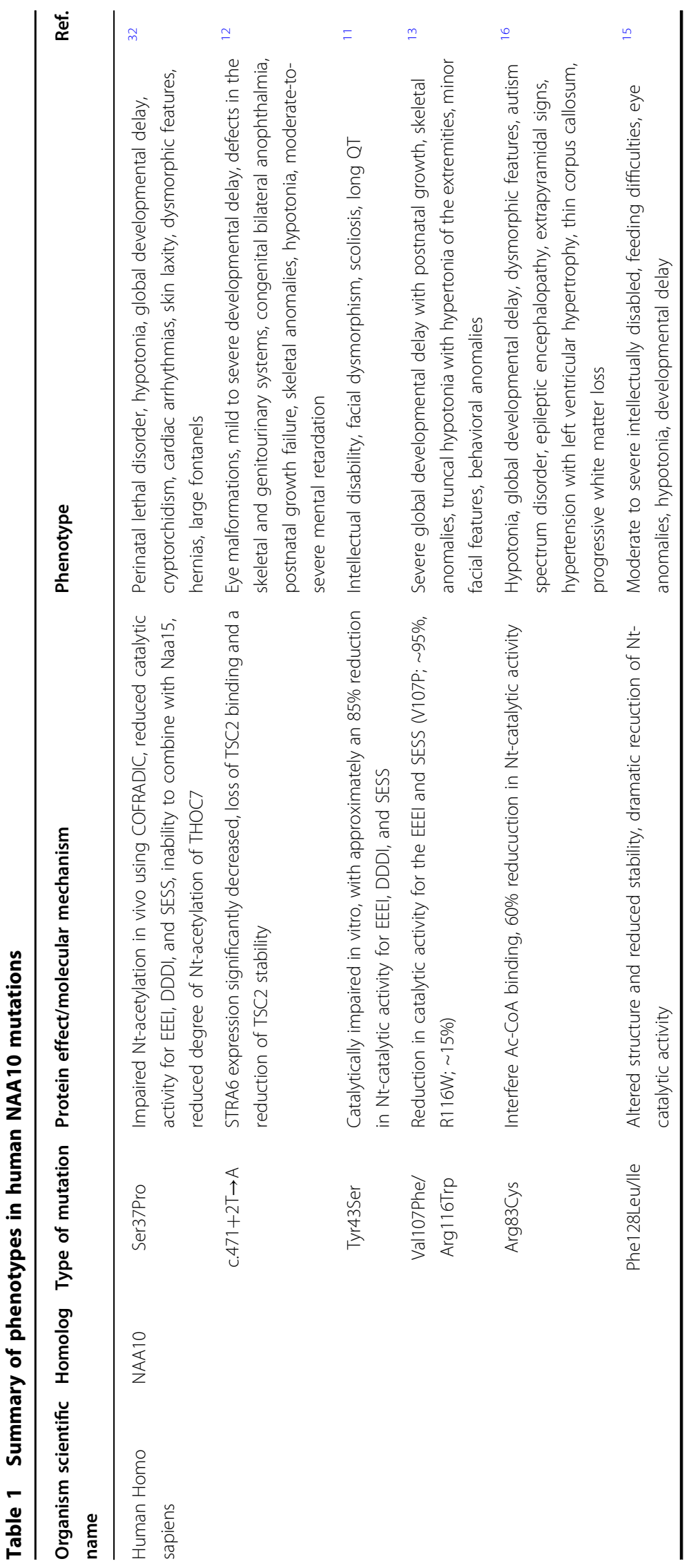




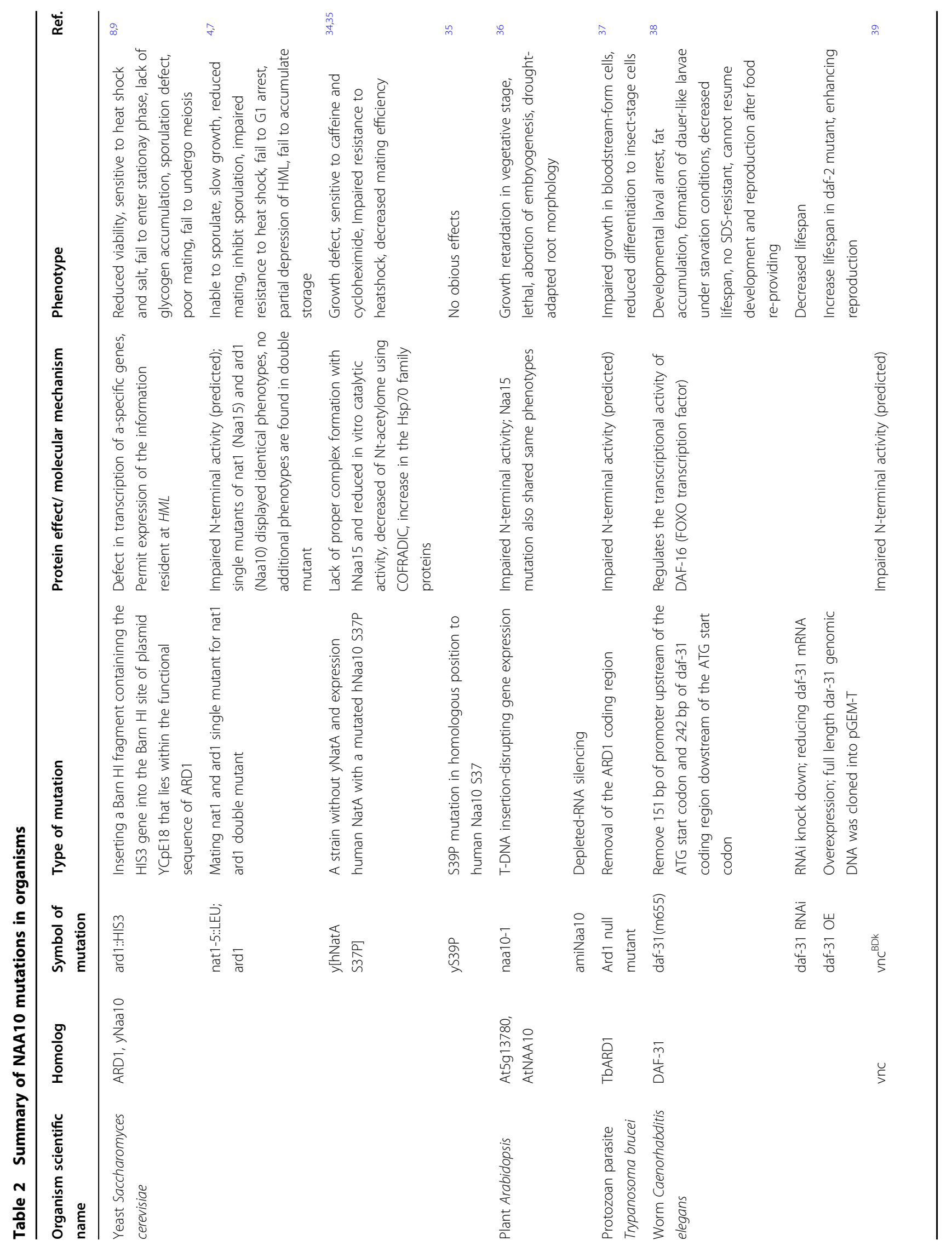


development. Furthermore, TSC2 is known to be stabilized by NAA10-dependent N-terminal acetylation. Cterminal truncated mutation of NAA10 demonstrates both loss of NAA10-TSC2 binding and reduced TSC2 protein levels, thereby resulting in perturbed mTOR signaling ${ }^{12}$. Given these findings, the N-terminal acetyltransferase activity of NAA10 plays a critical role in human development.

\section{Developmental roles in other organisms}

In several species, various developmental defects have also been observed due to a deficiency of NAA10 activity (Table 2). NAA10 was first discovered in yeast, where it was found to be crucial for cell growth and sporulation. The $y$ Naa 10 deleted strain, lacking NatA activity, is viable but exhibits a wide range of defects, including slow growth, de-repression of the silent mating type locus HML (Hidden MAT Left), temperature and salt sensitivity, and failure to enter G0 phase and sporulate ${ }^{4,7-9}$. Recently, the yeast model of Ogden syndrome expressing hNaa10 S37P in yNaa10 deficient strains has also been shown to have impaired growth and resistance to stress and mating ${ }^{34,35}$. Naa10 is required for Arabidopsis thaliana development, and thereby, Naa10 deficient mutations induce growth retardation and are lethal ${ }^{36}$. Additionally, the loss of Naa10 in Trypanosoma brucei leads to mortality $^{37}$. In Caenorhabditis elegans, daf-31 (the ortholog of NAA10) is essential for larval development, metabolism and adult lifespan. Daf-31 mutants fail to properly enter the dauer stage, which is pivotal for C. elegans survival during starvation (when nutrients are limited). Furthermore, the mutants display developmental arrest under abundant nutrition and shift their metabolism to fat accumulation $^{38}$. The importance of Naa10 in normal development is also supported by a Drosophila melanogaster study in which two hypomorphic mutations of Naa10 led to pleiotropic oogenesis defects, including aberrant mitoses, defects in egg chamber encapsulation and nurse cell chromatin dispersion ${ }^{39}$. Further, Naa10 deficient mutations have been shown to affect cell survival and proliferation and cause lethality. In a recent Danio rerio study, morpholino-mediated knockdown of Naa10 resulted in increased lethality, growth retardation and abnormal development, such as a bent axis, abnormal eyes, and bent tails, indicating the importance of Naa10 in early zebrafish development and viability ${ }^{40}$.

\section{Neuronal development}

During brain development in mouse, Naa10 and Naa15 (the auxiliary subunit of NatA) are highly expressed in regions rich in proliferating and migrating cells, such as the ventricular zone, neocortex, olfactory bulb, and hippocampus $^{41}$. The expression of both genes is downregulated as neurons differentiate and mitotic and 
migratory activities subside. Then, once again, their expression increases during postnatal development in the hippocampus and during the neuronal dendritic development of Purkinje cells (PCs) in the cerebellum. This finding indicates that the regulation of expression of both genes is related to neuronal development, especially in the hippocampus and in the PCs of the cerebellum.

The acetylation of $\alpha$-tubulin has been reported to be involved in regulating microtubule (MT) stability and dynamics, neuron polarization, and neurite branching and promotes vesicular transport on MTs in differentiated neurons ${ }^{42-46}$. A recent study showed that the inhibition of Naa10 or acetyltransferase activity significantly reduced the dendritic extension of cultured neurons ${ }^{47}$. Furthermore, Naa10 and Naa15 proteins co-localized with MTs in dendrites and induced the acetylation of tubulin in the brain fraction. Additionally, the dendritic arborization defect phenotype caused by the over-expression of HDAC6, a major deacetylase of $\alpha$-tubulin, was rescued by the co-expression of Naa10. Therefore, the authors postulated that Naa10 counteracts HDAC6 by acetylating $\alpha-$ tubulin, thereby promoting MT stability for dendritic development. In spite of this fact, it is not clear that $\alpha$ tubulin is the only distinct substrate of Naa10 that participates in dendritic development because the tubulin fractions contain both $\alpha / \beta$-tubulin. Moreover, it is uncertain whether tubulin is acetylated by the $\mathrm{N}$-terminal activity or lysine activity of Naa10. The lysine 40 residue of either tubulin is de-acetylated by HDAC6 ${ }^{48-50}$, so lysine acetylation may be a potential catalytic function of $\alpha$-tubulin. Characterizing the impact of whichever acetyl activity is involved in tubulin acetylation will help define a specific mechanism for neuronal development.

\section{Melanogenesis}

Naa10 knockdown in Zebra fish 2 days post-fertilization embryos showed less pigmentation, including abnormal development ${ }^{40}$. Pigmentation in organisms is due to the deposition of the pigment melanin, which is produced by specialized cells called melanocytes. In the process that produces melanin, which is called melanogenesis, the most important molecule among melanocyte-stimulating hormones (MSHs) is the proopiomelanocortin (POMC)derived peptide $\alpha$-MSH ${ }^{51,52}$. $\alpha$-MSH is known to be $\mathrm{N}$ terminally acetylated, and its stability and potency in stimulating pigment dispersion are increased by $\mathrm{Nt}$ acetylation $^{53-56}$. So far, $\mathrm{N}$-acetyl transferase enzyme acetylating $\alpha-\mathrm{MSH}$ has not been identified yet. NatA is known to acetylate the amino acids starting with Ser, Ala, Thr, Val, Gly, and $\mathrm{Cys}^{28}$. Based on this fact, the Nterminus of $\alpha-\mathrm{MSH}$, which starts with serine, demonstrates the possibility that $\alpha-\mathrm{MSH}$ could be a potential target of NatA. In the future, more studies are needed to reveal the direct regulation of $\alpha-\mathrm{MSH}$ and melanogenesis by Naa10.

\section{Spermatogenesis}

NAA11 (also known as Ard1b; ARD2), a homolog of $N A A 10$, is predominantly expressed in mouse ${ }^{57}$ and human $^{58}$ testis. Naa11 can reconstitute functional NAT in the presence of Naa15, where Naa11 is functionally equivalent to Naa10. Interestingly, NAA10 and NAA11 display opposite expression patterns during spermatogenesis. In the mouse, the expression of Naa11 is upregulated during meiosis, whereas Naa10 expression is downregulated. In contrast, Naa10 is expressed in premeiotic spermatogonia, which do not show Naa11 expression. Therefore, Pang et al. speculated that compensation for the loss of X-linked Naa10 occurs by expressing autosomal Naa11 due to the sex chromosome inactivation during male meiosis ${ }^{57,59}$. In this sense, they also suggested that the NAT activity of Naa10 could play a pivotal role in mitotic spermatogonia and that the NAT activity of Naa11 is crucial for post-meiotic male germ cells $^{59}$. Furthermore, the differential expression of NAA10 and NAA11 was shown in the human promyelocytic NB4 cell line upon differentiation ${ }^{21}$; NAA10 expression decreased with the induction of differentiation in NB4 cells, but the level of NAA11 remained unchanged, implying that Naa11 has a role in the cellular differentiation process and that Naa10 has a role in the cellular proliferation process. The differential expression pattern of Naa10/Naa11 suggests that Naa11 is complementary to Naa10 at least in the mice and that its biological role could be important in spermiogenesis or cellular processes $^{59}$.

\section{Lysine acetylation dependent functions of NAA10 during embryonic development}

Naa10 is known to regulate cellular processes, and its effects are not only catalyzed through its major activity as a NAT but also through the $\mathrm{N}-\varepsilon$-acetylation of several proteins ${ }^{2,3,60-73}$. The $\mathrm{N}-\varepsilon$-acetyl-activity of Naa10 requires auto-acetylation $^{2,67}$; this requirement is similar to that of other acetyltransferases, which acetylate themselves for their catalytic and functional activities ${ }^{74,75}$. Recently, gain or loss of function studies in mice have demonstrated that Naa10 plays an important role in osteoblast differentiation and the early phases of bone formation ${ }^{3}$. Overexpression of Naa10 in mice results in the delayed closure of calvarial fontanels and reduced bone density, osteoblast surfaces and mRNA levels of the osteoblastogenic genes in calvaria. In contrast, Naa10 deficient mice display calvarial and femoral bone development to a greater extent on postnatal day 3. Mechanically, Naa10 interacts with the RUNT domain of Runt-related transcription factor 2 (Runx2), which is the master regulator of 
osteoblast development, and acetylates it at K225. The lysine acetylation of Runx2 restricts its transcriptional activity by interfering with CBF $\beta$ binding to Runx2. On the other hand, Runx2 stabilizes Naa10 in osteoblasts during bone morphogenic protein 2 (BMP-2)-induced differentiation through its inhibition of IKK-mediated phosphorylation and degradation of Naa10, which in turn inhibits Runx $2^{3}$ (Table 3).

In addition to Runx2, the lysine acetyl-activity of Naa10 has been reported to target proteins such as $\beta$-catenin $(\mathrm{CTNNB} 1)^{60,61}$, Phosphoglycerate kinase 1 (PGK1) $^{72,73}$, Hypoxia inducible factor $1 \alpha(\mathrm{HIF}-1 \alpha)^{66-68}$, Myosin lightchain kinase $(\mathrm{MLCK})^{62}$, Androgen receptor(AR $)^{64,65}$, enzyme methionine sulfoxide reductase A (MSRA) ${ }^{63}$, SAM domain and HD domain containing protein 1 $(\mathrm{SAMHD} 1)^{69}$, Heat shock protein $70(\mathrm{Hsp} 70)^{70}$, Aurora kinase $\mathrm{A}(\mathrm{AuA})^{71}$, and Naa10 itself ${ }^{2,67}$. The lysine acetyltransferase activity of NAA10 catalyzing these targets has not yet been directly implicated in development, but the targets are essential for developmental signaling pathways, such as Wnt/ $\beta$-catenin signaling ${ }^{76-78}$, autophagy $^{79-81}$ and the HIF-1 regulatory pathway ${ }^{82-84}$. Accordingly, there is a possibility that NAA10 could influence developmental processes via these signaling pathways. On the other hand, Magin et al. reported data on the lysine acetyl-activity of NAA10, demonstrating that NAA10 does not acetylate the lysine residues of MLCK, MSRA, or RUNX2 ${ }^{85}$. For the lysine acetyl-activity of NAA10, earlier reports demonstrated that the autoacetylation of NAA10 at K136 was a critical step to generate an active form. For example, NAA10 K136R, a mutation in the auto-acetylation site, failed to acetylate $\beta$ catenin and did not succeed in the recruitment of $\beta$ catenin on the cyclin D1 promoter $^{2}$. Therefore, the inability of NAA10 to acetylate the lysine residues of MLCK, MSRA and RUNX2 could be due to non-auto acetylated NAA10. There is a possibility that Magin et al. performed their assay with non-auto acetylated NAA10, thus losing lysine acetyl-activity in vitro. Nevertheless, more research is required to understand the role of lysine acetylation by Naa10.

\section{Acetylation independent functions of NAA10 during embryonic development}

Several recent studies have suggested that NAA10 is able to interact directly with other proteins and enhance or inhibit the activity of its partner in an acetylationindependent manner ${ }^{86}$. The signaling pathways of NAA10 and its binding partners have been actively investigated for cell growth and function in cancer research, but reports on acetylation-independent roles of NAA10 during development are very partial as described below. 
Genomic imprinting regulation in embryonic development

Genomic imprinting is an epigenetic process controlled by DNA methylation, and it plays a vital role in normal development. During embryonic development, the establishment of appropriate imprinting is accomplished by the intact regulation of Dnmt3 and Dnmt1 DNA methyltransferases ${ }^{87}$. Lee et al. reported that Naa10 deficiency mice exhibited developmental defects, including partial embryonic lethality, postnatal growth retardation, brain disorders, and maternal effect lethality, resembling the phenotypes caused by the dysregulation of genomic imprinting ${ }^{88}$ (Table 3). The authors described that despite questions about how Naa10 selectively binds to the imprinted allele, mechanistically, Naa10 binds to the unmethylated GCXGXG in the imprinting control region (ICR)/differentially methylated region (DMR) of the imprinted allele and then recruits Dnmt1 for methylation in the $S$ phase. In addition, by showing the disrupted DNA binding activity of clinical NAA10 mutations (S37P, V107F, and R116W) to ICR, a potential connection between NAA10 mutation-associated syndromes and defects in DNA methylation and genomic imprinting was presented. Together, these results suggested that normal DNA methylation and genomic imprinting is regulated by appropriate ICR binding of Naa10 and DNMT1 recruitment during development. Previously, Lee et al. demonstrated that Naa10 acetyl activity does not mediate Naa10DNMT1 binding and does not stimulate the activity of DNMT1 ${ }^{89}$. However, even though Naa10 acetyl activity is dispensable for stimulating DNMT1 activity, we could obtain an insight from their recent study that showed that the N-terminal acetyl-activity of Naa10 is required for binding to ICRs/DMRs. The authors showed that reduced DNA binding to clinically relevant mutations (S37P; defects in Naa15 binding domain for NatA complex, V107F; mutation in the acetyltransferase domain, R116W; putative interference with Ac-CoA binding ${ }^{15}$ ) are associated with $\mathrm{N}$-terminal acetyltransferase activity, which is not related to the DNA-binding domain at the $\mathrm{C}$ terminus of Naa10 $0^{9}$ Given these results, there is a possibility that the enzyme activity of Naa10 directly or indirectly influences ICR binding. For example, Naa10 may combine with other proteins to acetylate the $\mathrm{N}$-terminal residue, and then, these proteins may interact with ICRs/DMRs together. Interestingly, unlike other reports in other species, including humans, Naa10 deficient mice exhibited only a few phenotypes, such as embryonic lethality and growth retardation ${ }^{88}$. Moreover, some of the Naa10 deficient mice developed to term. The authors suggested some reasons for the decreased penetrance of various phenotypes. First, they suggested that this finding was a general effect of imprinting disorders caused by the deficiency of genes that maintain genomic imprinting during global DNA demethylation in zygotes. Second, the remaining DNA methylation occurred at different regions in each individual mouse, which may contribute to stochastic phenotypes. Third, the dysregulation of other imprinted genes, such as Igf2, Ascl2, and Grb10, and the effects of unidentified non-imprinted genes or protein acetylation by Naa10 deficiency are suggested. In spite of these descriptions, there are still issues to be resolved. It would have been a more complete study if the maternal effects leading to the death of embryos and neonatal mice were explained and if a histological analysis in not only placenta but also whole embryos was performed to determine the precise effects of embryonic lethality. Therefore, more elaborate mechanisms remain to be determined and more extensive mouse studies are needed.

\section{Vasculogenesis}

$\mathrm{Xu}$ et al. demonstrate a potential role of Naa10 in vasculogenesis and neurogenesis through the regulation of UNC-5 Homolog B (UNC5B) and Netrin-1 (NTA1) ${ }^{90}$. UNC5B and its ligand NTA1 function as essential genes in morphogenesis of the vascular system or nervous system and the negative regulation of Naa10. Knockdown of Naa10 induced morphological changes (shuttle-shaped membrane protrusion) and increased the ability for tube formation in both H1299 lung cancer cell lines and immortalized mouse endothelial cell lines. Additionally, the expression of UNC5B and NTA1 increased in Naa10 knockdown $\mathrm{H} 1299$ cells, and negative regulation was also verified in the mouse caudal half region of E10 embryos (when exuberant vasculature developed at that stage) ${ }^{90}$. However, a direct mechanism explaining how Naa10 regulates NTA1 and its receptor UNC5B has not been determined. Nevertheless, it has been reported that Naa10 physically binds with RelA/p65, an NFkB transcription factor and a negative regulator of NTA ${ }^{91,92}$. Therefore, the authors speculate that Naa10 physically interacts with p65, thereby negatively regulating NTA1 and its receptor UNC5B.

\section{Conclusion and perspectives}

In this review, we summarized the functions of NAA10 that are enzyme activity-dependent and -independent during the development of several organisms. Most studies have focused on the N-terminal activity of NAA10, whereas some findings have been related to lysine activity and the independent role of NAA10. Our overall understanding of the function of NAA10 in developmental processes suggests the following insights. First, depending on which acetyl-activity is acting and how it affects development, the corresponding physiological responses could be very different. NatA is presumed to act on a large number of substrates involved in a multitude of different cellular processes. As NatA targets many substrates, it could be expected to affect diverse cellular processes and 
eventually lead to developmental processes in many different organs. On the other hand, since the known targets of lysine acetylation by NAA10 mediate important signaling pathways during development, more intensive and accurate mechanistic studies of these signaling pathways could reveal the role of NAA10 in development. It is also important to understand how the regulation of NAA10 itself is managed during the developmental stage. For example, NAA10 auto-acetylation activates its lysine catalytic activity, and active IKK $\beta$ degrades NAA10 by phosphorylation $^{91}$. Furthermore, NAA10 acts as a coactivator of DNMT1 without its enzymatic activity. Likewise, given that NAA10 carries out a wide spectrum of functions, the cellular-, tissue- and developmental stagespecific effects of NAA10 need to be discovered. Second, interestingly, some individuals normally survive and grow with decreased or mutated NAA10, which suggests the possibility of a homologous gene that might compensate for the function of NAA10. This suggestion is supported by the existence of $N A A 11$, which has been identified as a homologous gene of NAA10, and the reciprocal expression between NAA10 and NAA11 during spermatogenesis. However, we are unable to answer whether NAA11, whose expression appears to be limited to the testis, can cover the entire function of the ubiquitously expressed NAA10 protein. Therefore, an open question about the existence of another homologous gene to NAA1O in different tissues remains. The goal of future studies will be to elaborate on the cellular/molecular specific activity and distinct pathways of NAA10. Above all, in vivo studies are necessary to analyze the definite biological effects of NAA10.

\section{Acknowledgements}

This work was supported by a National Research Foundation of Korea (NRF) grant funded by the Korean Government (NRF-2012R1A3A2026454, NRF2012R1A6A3A04040206, NRF-2015M3A9B6029138, and NRF2017R1D1A1B03032286).

\section{Conflict of interest}

The authors declare that they have no conflict of interest.

\section{Publisher's note}

Springer Nature remains neutral with regard to jurisdictional claims in published maps and institutional affiliations.

Received: 28 March 2018 Accepted: 11 April 2018

Published online: 27 July 2018

\footnotetext{
References

1. Park, E. C. \& Szostak, J. W. ARD1 and NAT1 proteins form a complex that has Nterminal acetyltransferase activity. EMBO J. 11, 2087-2093 (1992).

2. Seo, J. H. et al. Arrest defective 1 autoacetylation is a critical step in its ability to stimulate cancer cell proliferation. Cancer Res. 70, 4422-4432 (2010).

3. Yoon, $H$. et al. NAA10 controls osteoblast differentiation and bone formation as a feedback regulator of Runx2. Nat. Commun. 5, 5176 (2014).
}

4. Mullen, J. R. et al. Identification and characterization of genes and mutants for an N-terminal acetyltransferase from yeast. EMBO J. 8, 2067-2075 (1989).

5. Kuo, H. P. et al. ARD1 stabilization of TSC2 suppresses tumorigenesis through the mTOR signaling pathway. Sci. Signal. 3, ra9 (2010).

6. Eiyama, A. \& Okamoto, K. Protein N-terminal acetylation by the NatA complex is critical for selective mitochondrial degradation. J. Biol. Chem. 290, 25034-25044 (2015)

7. Lee, F. J., Lin, L. W. \& Smith, J. A. N alpha acetylation is required for normal growth and mating of Saccharomyces cerevisiae. J. Bacteriol. 171, 5795-5802 (1989).

8. Whiteway, M., Freedman, R., Van Arsdell, S., Szostak, J. W. \& Thorner, J. The yeast ARD1 gene product is required for repression of cryptic mating-type information at the HML locus. Mol. Cell. Biol. 7, 3713-3722 (1987).

9. Whiteway, M. \& Szostak, J. W. The ARD1 gene of yeast functions in the switch between the mitotic cell cycle and alternative developmental pathways. Cell 43(2 Pt 1), 483-492 (1985).

10. Dorfel, M. J. \& Lyon, G. J. The biological functions of Naa10 - from aminoterminal acetylation to human disease. Gene 567, 103-131 (2015).

11. Casey, J. P. et al. NAA10 mutation causing a novel intellectual disability syndrome with Long QT due to N-terminal acetyltransferase impairment. Sci. Rep. 5, 16022 (2015).

12. Esmailpour, T. et al. A splice donor mutation in NAA10 results in the dysregulation of the retinoic acid signalling pathway and causes Lenz microphthalmia syndrome. J. Med. Genet. 51, 185-196 (2014).

13. Popp, B. et al. De novo missense mutations in the NAA10 gene cause severe non-syndromic developmental delay in males and females. Eur. J. Hum. Genet. 23, 602-609 (2015).

14. Rope, A. F. et al. Using VAAST to identify an X-linked disorder resulting in lethality in male infants due to $\mathrm{N}$-terminal acetyltransferase deficiency. Am. J. Hum. Genet. 89, 28-43 (2011).

15. Saunier, $\mathrm{C}$. et al. Expanding the phenotype associated with NAA10-related Nterminal acetylation deficiency. Hum. Mutat. 37, 755-764 (2016).

16. Sidhu, M., Brady, L., Tarnopolsky, M. \& Ronen, G. M. Clinical manifestations associated with the N-terminal-acetyltransferase NAA10 gene mutation in a girl: ogden syndrome. Pediatr. Neurol. 76, 82-85 (2017).

17. Tribioli, C. et al. Isolation of new genes in distal Xq28: transcriptional map and identification of a human homologue of the ARD1 N-acetyl transferase of Saccharomyces cerevisiae. Hum. Mol. Genet. 3, 1061-1067 (1994).

18. Chun, K. H. et al. Differential regulation of splicing, localization and stability of mammalian ARD1235 and ARD1225 isoforms. Biochem. Biophys. Res. Commun. 353, 18-25 (2007).

19. Kim, S. H. et al. Characterization of ARD1 variants in mammalian cells. Biochem. Biophys. Res. Commun. 340, 422-427 (2006).

20. Seo, J. H., Park, J. H., Lee, E. J. \& Kim, K. W. Different subcellular localizations and functions of human ARD1 variants. Int. J. Oncol. 46, 701-707 (2015).

21. Arnesen, $\mathrm{T}$. et al. Characterization of hARD2, a processed hARD1 gene duplicate, encoding a human protein N-alpha-acetyltransferase. BMC Biochem. 7, 13 (2006).

22. Xue, L. et al. Global expression profiling reveals genetic programs underlying the developmental divergence between mouse and human embryogenesis. BMC Genomics 14, 568 (2013).

23. $\mathrm{Yi}, \mathrm{H}$. et al. Gene expression atlas for human embryogenesis. FASEB J. 24, 3341-3350 (2010).

24. Noguchi, S. et al. FANTOM5 CAGE profiles of human and mouse samples. Sci. Data 4, 170112 (2017).

25. Consortium, F. et al. A promoter-level mammalian expression atlas. Nature 507, 462-470 (2014).

26. Andersson, R. et al. An atlas of active enhancers across human cell types and tissues. Nature 507, 455-461 (2014).

27. Yu, N. Y. et al. Complementing tissue characterization by integrating transcriptome profiling from the Human Protein Atlas and from the FANTOM5 consortium. Nucleic Acids Res. 43, 6787-6798 (2015).

28. Arnesen, $\mathrm{T}$. Towards a functional understanding of protein N-terminal acetylation. PLoS Biol. 9, el001074 (2011).

29. Aksnes, H., Hole, K. \& Arnesen, T. Molecular, cellular, and physiological significance of N-terminal acetylation. Int Rev. Cell Mol. Biol. 316, 267-305 (2015).

30. Arnesen, T. et al. Proteomics analyses reveal the evolutionary conservation and divergence of $\mathrm{N}$-terminal acetyltransferases from yeast and humans. Proc. Natl Acad. Sci. USA 106, 8157-8162 (2009)

31. Driessen, H. P., de Jong, W. W., Tesser, G. I. \& Bloemendal, H. The mechanism of N-terminal acetylation of proteins. CRC Crit. Rev. Biochem. 18, 281-325 (1985). 
32. Myklebust, L. M. et al. Biochemical and cellular analysis of Ogden syndrome reveals downstream Nt-acetylation defects. Hum. Mol. Genet. 24, 1956-1976 (2015).

33. Myklebust, L. M., Stove, S. I. \& Arnesen, T. Naa10 in development and disease. Oncotarget 6, 34041-34042 (2015).

34. Van Damme, P., Stove, S. I., Glomnes, N., Gevaert, K. \& Arnesen, T. A Saccharomyces cerevisiae model reveals in vivo functional impairment of the Ogden syndrome N-terminal acetyltransferase NAA10 Ser37Pro mutant. Mol. Cell. Proteom. 13, 2031-2041 (2014).

35. Dorfel, M. J. et al. Proteomic and genomic characterization of a yeast model for Ogden syndrome. Yeast 34, 19-37 (2017).

36. Linster, E. et al. Downregulation of N-terminal acetylation triggers ABA-mediated drought responses in Arabidopsis. Nat. Commun. 6, 7640 (2015).

37. Ingram, A. K., Cross, G. A. \& Horn, D. Genetic manipulation indicates that ARD1 is an essential N(alpha)-acetyltransferase in Trypanosoma brucei. Mol. Biochem. Parasitol. 111, 309-317 (2000).

38. Chen, D. et al. daf-31 encodes the catalytic subunit of $\mathrm{N}$ alphaacetyltransferase that regulates Caenorhabditis elegans development, metabolism and adult lifespan. PLoS Genet 10, e1004699 (2014).

39. Wang, Y. et al. Drosophila variable nurse cells encodes arrest defective 1 (ARD1), the catalytic subunit of the major $\mathrm{N}$-terminal acetyltransferase complex. Dev. Dyn. 239, 2813-2827 (2010).

40. Ree, R. et al. The N-terminal acetyltransferase Naa10 is essential for zebrafish development. Biosci. Rep. 35, pii. e00249 (2015).

41. Sugiura, N., Adams, S. M. \& Corriveau, R. A. An evolutionarily conserved Nterminal acetyltransferase complex associated with neuronal development. J. Biol. Chem. 278, 40113-40120 (2003).

42. Li, W. et al. Sirtuin 2, a mammalian homolog of yeast silent information regulator-2 longevity regulator, is an oligodendroglial protein that decelerates cell differentiation through deacetylating alpha-tubulin. J. Neurosci. 27, 2606-2616 (2007).

43. North, B. J., Marshall, B. L., Borra, M. T., Denu, J. M. \& Verdin, E. The human Sir2 ortholog, SIRT2, is an NAD+-dependent tubulin deacetylase. Mol. Cell 11, 437-444 (2003).

44. Ohkawa, N., Fujitani, K., Tokunaga, E., Furuya, S. \& Inokuchi, K. The microtubule destabilizer stathmin mediates the development of dendritic arbors in neuronal cells. J. Cell Sci. 120(Pt 8), 1447-1456 (2007).

45. Vaillant, A. R. et al. Signaling mechanisms underlying reversible, activitydependent dendrite formation. Neuron 34, 985-998 (2002).

46. MacRae, T. H. Tubulin post-translational modifications--enzymes and their mechanisms of action. Eur. J. Biochem 244, 265-278 (1997).

47. Ohkawa, N. et al. N-acetyltransferase ARD1-NAT1 regulates neuronal dendritic development. Genes Cells 13, 1171-1183 (2008).

48. Hubbert, C. et al. HDAC6 is a microtubule-associated deacetylase. Nature 417, 455-458 (2002).

49. Matsuyama, A. et al. In vivo destabilization of dynamic microtubules by HDAC6-mediated deacetylation. EMBO J. 21, 6820-6831 (2002).

50. Zhang, Y. et al. HDAC-6 interacts with and deacetylates tubulin and microtubules in vivo. EMBO J. 22, 1168-1179 (2003).

51. Pritchard, L. E. et al. Proopiomelanocortin-derived peptides in rat cerebrospinal fluid and hypothalamic extracts: evidence that secretion is regulated with respect to energy balance. Endocrinology 144, 760-766 (2003).

52. Geschwind, I. I., Huseby, R. A. \& Nishioka, R. The effect of melanocytestimulating hormone on coat color in the mouse. Recent Prog. Horm. Res. $\mathbf{2 8}$ 91-130 (1972).

53. O'Donohue, T. L., Handelmann, G. E., Chaconas, T., Miller, R. L. \& Jacobowitz, D. $\mathrm{M}$. Evidence that $\mathrm{N}$-acetylation regulates the behavioral activity of alpha-MSH in the rat and human central nervous system. Peptides 2, 333-344 (1981).

54. Glembotski, C. C. Acetylation of alpha-melanotropin and beta-endorphin in the rat intermediate pituitary. Subcellular localization. J. Biol. Chem. 257, 10493-10500 (1982)

55. O'Donohye, T. L., Handelmann, G. E., Miller, R. L. \& Jacobowitz, D. M. Nacetylation regulates the behavioral activity of alpha-melanotropin in a multineurotransmitter neuron. Science 215, 1125-1127 (1982).

56. Wilkinson, C. W. Roles of acetylation and other post-translational modifications in melanocortin function and interactions with endorphins. Peptides 27, 453-471 (2006)

57. Pang, A. L. et al. Characterization, and expression analysis of the novel acetyltransferase retrogene Ard1b in the mouse. Biol. Reprod. 81, 302-309 (2009).
58. Pang, A. L., Clark, J., Chan, W. Y. \& Rennert, O. M. Expression of human NAA1 1 (ARD1B) gene is tissue-specific and is regulated by DNA methylation. Epigenetics 6, 1391-1399 (2011).

59. Pang A, Rennert O. Protein acetylation and spermatogenesis. Reprod. Syst. Sex Disord. Suppl 1: 5 (2013).

60. Lim, J. H., Chun, Y. S. \& Park, J. W. Hypoxia-inducible factor-1alpha obstructs a Wnt signaling pathway by inhibiting the hARD1-mediated activation of betacatenin. Cancer Res. 68, 5177-5184 (2008).

61. Lim, J. H., Park, J. W. \& Chun, Y. S. Human arrest defective 1 acetylates and activates beta-catenin, promoting lung cancer cell proliferation. Cancer Res. 66, 10677-10682 (2006).

62. Shin, D. H., Chun, Y. S., Lee, K. H., Shin, H. W. \& Park, J. W. Arrest defective-1 controls tumor cell behavior by acetylating myosin light chain kinase. PLoS ONE 4, e7451 (2009).

63. Shin, S. H. et al. Arrest defective 1 regulates the oxidative stress response in human cells and mice by acetylating methionine sulfoxide reductase A. Cell Death Dis. 5, e1490 (2014)

64. Wang, Z. et al. Inactivation of androgen-induced regulator ARD1 inhibits androgen receptor acetylation and prostate tumorigenesis. Proc. Natl Acad. Sci. USA 109, 3053-3058 (2012).

65. DePaolo, J. S. et al. Acetylation of androgen receptor by ARD1 promotes dissociation from HSP90 complex and prostate tumorigenesis. Oncotarget 7, 71417-71428 (2016).

66. Lee, M. N. et al. Roles of arrest-defective protein 1(225) and hypoxia-inducible factor 1alpha in tumor growth and metastasis. J. Natl Cancer Inst. 102, 426-442 (2010).

67. Seo, J. H. et al. Autoacetylation regulates differentially the roles of ARD1 variants in tumorigenesis. Int J. Oncol. 46, 99-106 (2015).

68. Jeong, J. W. et al. Regulation and destabilization of HIF-1alpha by ARD1mediated acetylation. Cell 111, 709-720 (2002).

69. Lee, E. J. et al. SAMHD1 acetylation enhances its deoxynucleotide triphosphohydrolase activity and promotes cancer cell proliferation. Oncotarget $\mathbf{8}$, 68517-68529 (2017)

70. Seo, J. H. et al. ARD1-mediated Hsp70 acetylation balances stress-induced protein refolding and degradation. Nat. Commun. 7, 12882 (2016).

71. Vo, T. T. L. et al. ARD1-mediated aurora kinase A acetylation promotes cell proliferation and migration. Oncotarget 8, 57216-57230 (2017).

72. Qian, X. et al. Phosphoglycerate kinase 1 phosphorylates beclin1 to induce autophagy. Mol. Cell 65, 917-931 e6 (2017).

73. Qian, X., Li, X. \& Lu, Z. Protein kinase activity of the glycolytic enzyme PGK1 regulates autophagy to promote tumorigenesis. Autophagy 13, 1246-1247 (2017).

74. Santos-Rosa, H., Valls, E., Kouzarides, T. \& Martinez-Balbas, M. Mechanisms of P/ CAF auto-acetylation. Nucleic Acids Res. 31, 4285-4292 (2003).

75. Mellert, H. S. \& McMahon, S. B. Biochemical pathways that regulate acetyltransferase and deacetylase activity in mammalian cells. Trends Biochem. Sci. 34, 571-578 (2009).

76. Basson, M. A. Signaling in cell differentiation and morphogenesis. Cold Spring Harb. Perspect. Biol. 4, pii. a008151 (2012).

77. MacDonald, B. T., Tamai, K. \& He, X. Wnt/beta-catenin signaling: components, mechanisms, and diseases. Dev. Cell 17, 9-26 (2009).

78. Clevers, H. Wnt/beta-catenin signaling in development and disease. Cell 127, 469-480 (2006)

79. Mizushima, N. Autophagy: process and function. Genes Dev. 21, 2861-2873 (2007).

80. Cecconi, F. \& Levine, B. The role of autophagy in mammalian development: cell makeover rather than cell death. Dev. Cell 15, 344-357 (2008).

81. Yue, Z., Jin, S., Yang, C., Levine, A. J. \& Heintz, N. Beclin 1, an autophagy gene essential for early embryonic development, is a haploinsufficient tumor suppressor. Proc. Natl Acad. Sci. USA 100, 15077-15082 (2003).

82. Tomita, S. et al. Defective brain development in mice lacking the Hif-1alpha gene in neural cells. Mol. Cell Biol. 23, 6739-6749 (2003).

83. lyer, N. V. et al. Cellular and developmental control of $\mathrm{O} 2$ homeostasis by hypoxia-inducible factor 1 alpha. Genes Dev. 12, 149-162 (1998).

84. Semenza, G. L. Angiogenesis in ischemic and neoplastic disorders. Annu. Rev. Med. 54, 17-28 (2003)

85. Magin, R. S., March, Z. M. \& Marmorstein, R. The N-terminal acetyltransferase Naa10/ARD1 does not acetylate lysine residues. J. Biol. Chem. 291, 5270-5277 (2016).

86. Hollebeke, J., Van Damme, P. \& Gevaert, K. N-Terminal acetylation and other functions of Na-acetyltransferases. Biol. Chem. 393, 291-298 (2012). 
87. Barlow, D. P. \& Bartolomei, M. S. Genomic imprinting in mammals. Cold Spring Harb. Perspect. Biol. 6, a018382 (2014).

88. Lee, C. C. et al. The Role of N-alpha-acetyltransferase 10 protein in DNA methylation and genomic imprinting. Mol. Cell 68, 89-103 e7 (2017).

89. Lee, C. F. et al. hNaa10p contributes to tumorigenesis by facilitating DNMT1-mediated tumor suppressor gene silencing. J. Clin. Invest. 120, 2920-2930 (2010).
90. Xu, H., Han, Y., Liu, B. \& Li, R. Unc-5 homolog B (UNC5B) is one of the key downstream targets of N-alpha-Acetyltransferase 10 (Naa10). Sci. Rep. 6, 38508 (2016).

91. $\mathrm{Xu}, \mathrm{H}$. et al. N-alpha-acetyltransferase 10 protein inhibits apoptosis through RelA/p65-regulated MCL1 expression. Carcinogenesis 33, 1193-1202 (2012).

92. Mirakaj, V. et al. Netrin-1 dampens pulmonary inflammation during acute lung injury. Am. J. Respir. Crit. Care Med. 181, 815-824 (2010). 